\title{
An excess of perfection
}

The good news is that the three-degree cosmic microwave background has the exact spectrum of black-body radiation, and is spatially featureless. The bad news is the same.

\section{Crystal Clty, VIrgInla}

EVERY now and then, data from balloonand rocket-borne experiments have suggested that the spectrum of the threedegree background, the diluted remains of the Big Bang itself, departs a little from Planck's simple formula for radiation from a body of constant temperature. Such an indication two years ago, from the Berkeley-Nagoya collaboration, set off a spate of speculations: perhaps the reported excess of photons on the infrared side of the $2.7-\mathrm{K}$ peak was the remnant of some fierce process of early cosmic history, connected with the formation of galaxies; perhaps it even signalled some fundamental flaw in Big-Bang cosmology.

The most remarkable finding delivered to the annual meeting of the American Astronomical Society last week was the revelation that $\mathrm{COBE}$, the Cosmic Background Explorer satellite, has laid to rest, apparently forever, all such speculations. Less than two months into its year-long mission, COBE has given to project scientist John Mather of the NASA/Goddard Space Flight Center data that allowed him to draw a graph in which about twenty measurements of the absolute intensity of the microwave background at twenty different frequencies fall precisely on a Planck curve for black-body radiation at a temperature of $2.735 \pm 0.06 \mathrm{~K}$. These measurements, whose 1 per cent uncertainty will be reduced by at least an order of magnitude by the end of the year, leave the Berkeley-Nagoya excess high and dry.

That was the good news. The demise of the Berkeley-Nagoya excess was generally welcomed because even remotely plausible explanations for it had been hard to come by. Cosmologists were obliged to suppose, in one example, that there might have been an early pregalactic generation of supermassive stars, which had burned brightly but briefly before exploding as gigantic supernovae. The radiation from these stars was then to be thermalized by dust grains formed from material ejected by the supernovae. The net result of this intricate scheme was to have been an excess of radiation sitting just next to the peak of the microwave background.

Few will be sorry to say goodbye to such convoluted theories. Nevertheless, the thought that the microwave background should be perfectly thermal is not an entirely happy one. Galaxy formation, however it happens, cannot be done secretly. Galaxies may arise from the growth of primordial density fluctuations, by the aggregation of material around loops of cosmic string, or through the triggering action of shock waves from giant supernovae, but no matter what the route, a more or less uniform distribution of material must gather together in gravitationally bound clumps, and this can happen only if gravitational potential energy is somehow disposed of.

Inevitably, as it collapses into discrete objects, gas will heat up and begin to shine; this energy redshifted to lower frequencies, must still lurk somewhere in the infrared or radio region of the cosmic spectrum. If COBE continues to see an apparently pristine spectrum of cosmic radiation, unmodified by any subsequent

\section{SN1987A levels off}

ONE year after it exploded, supernova 1987A emitted its first gamma rays. One year later again, the notorious half-millisecond pulsar appeared and disappeared. Now, with its third anniversary coming up next month, SN1987A has revealed itself a little more. In IAU Circular 4933, astronomers from the European Southern Observatory say that the supernova's total brightness, which had been falling exponentially, has at last levelled out, indicating the presence of an internal energy source giving out about $10^{38} \mathrm{erg} \mathrm{s}^{-1}$.

As the exponential light curve, powered by ${ }^{57} \mathrm{Co}$ synthesized in the explosion, dwindled, astronomers had been anxiously waiting for heat provided by the accretion of material onto the remnant neutron star to make itself known. The latest observations, from P. Bouchet, I.J. Danziger and L.B. Lucy, show that the infrared magnitude of SN1987A has remained essentially constant since late last year.

But this is not a confirmation that the transient submillisecond pulsar was really there. According to Stan Woosley of Santa Cruz, the estimated magnitude is suggestive of a non-pulsing neutron star radiating at the Eddington limit, the maximum it can generate without blowing the accreting material away. And even if a pulsar were now found, it would not necessarily confirm the reality of the submillisecond pulsar, whose energy output and spindown rate were so poorly determined that it is impossible to guess what it might look like a year later.

D.L. energy releases, cosmologists will be forced to make their galaxies in an increasingly furtive way.

The difficulty is exacerbated by a second early result from COBE.George Smoot, of the University of California at Berkeley, revealed that another of the detectors on the satellite had so far failed to find any irregularities in the spatial distribution of the cosmic background radiation. This adds to a long litany of negative results from searches for fluctuations in the intensity of the background from one point in the sky to another. A map of these intensity fluctuations is in effect a map of the density of the Universe at an age of about 300,000 years, when the photons observed now by COBE last interacted with matter.

But in a completely uniform Universe, galaxies could never appear. Whether galaxies themselves or massive stars form first, or even if cosmic strings pull matter around, some fluctuations in density must have been present. Because there are galaxies now, in other words, there must also be non-uniformities in the microwave background - at some level and on some angular scale.

So far, nobody has found any irregularities on any scale, and COBE's preliminary data reduce the upper limit on fluctuations at an angular scale of $7^{\circ}$ by about a factor of two. Another factor of ten improvement should come when COBE has mapped the sky in full.

Theoretical cosmologists have been enormously ingenious, of course, in modifying their ideas of galaxy formation to stay beneath the upper limits given to them by their observational colleagues. But the limits continue to descend, and the finetuning of the theories seems to be stretched almost to breaking point. And even in current theories, galaxy formation tends to proceed from initial conditions found by assertion through the medium of so many hypothetical types of dark matter that many find the whole business unappealing.

If, by the Big Bang, one means the notion of a universe expanding smoothly from a hot initial singularity, then COBE's first results are a glorious confirmation. But if one also includes twenty years' worth of sophisticated work on galaxy formation as an integral part of the Big-Bang model, then cosmologists could be in for an upsetting decade.

David LIndley 